\title{
The mechanisms of Chuanxiong Rhizoma in treating spinal cord injury based on network pharmacology and experimental verification
}

\author{
Bo Tao $^{1,2,3 \#}$, Qi Wang ${ }^{1,2,3 \#}$, Jiangang Cao ${ }^{1,2,3 \#}$, Yimingjiang Yasen ${ }^{1,2,3 \#}$, Lei Ma ${ }^{1,2,3}$, Chao Sun ${ }^{1,2,3}$, \\ Jun Shang ${ }^{1,2,3}$, Shiqing Feng ${ }^{1,2,3}$
}

${ }^{1}$ Department of Orthopedics, Tianjin Medical University General Hospital, Tianjin, China; ${ }^{2}$ Tianjin Key Laboratory of Spine and Spinal Cord Injury, Tianjin, China; ${ }^{3}$ International Science and Technology Cooperation Base of Spinal Cord Injury, Tianjin, China

Contributions: (I) Conception and design: B Tao, S Feng; (II) Administrative support: S Feng (III) Provision of study materials or patients: B Tao, S Feng; (IV) Collection and assembly of data: B Tao, Q Wang, J Cao, S Feng; (V) Data analysis and interpretation: B Tao, Q Wang, J Cao; (VI) Manuscript writing: All authors; (VII) Final approval of manuscript: All authors.

\#These authors contributed equally to this work.

Correspondence to: Shiqing Feng. International Science and Technology Cooperation Base of Spinal Cord Injury, Tianjin Key Laboratory of Spine and Spinal Cord, Department of Orthopedics, Tianjin Medical University General Hospital, 154 Anshan Road, Heping District, Tianjin 300052, China. Email: sqfeng@tmu.edu.cn.

Background: Chuanxiong Rhizoma (CR) is a common traditional Chinese medicine (TCM) that has been widely used in the treatment of spinal cord injury (SCI). However, the underlying molecular mechanism of CR is still largely unknown. This study was designed to explore the bioactive components and the mechanism of CR in treating SCI based on a network pharmacology approach and experimental validation.

Methods: First, the active compounds and related target genes in CR were screened from the Traditional Chinese Medicine Systems Pharmacology (TCMSP) database. Subsequently, the corresponding target genes of SCI were collected by the Therapeutic Target Database (TTD) and GeneCards database. A protein-protein interaction (PPI) network was constructed using the STRING database. Furthermore, GO function and KEGG enrichment analysis of the targets were analyzed using DAVID tools. Subsequently, the AutoDock software for molecular docking was adopted to verify the above network pharmacology analysis results between the active components and key targets. Finally, an SCI rat model animal validation experiment was assessed to verify the reliability of the network pharmacology results.

Results: There were 7 active ingredients identified in CR and 246 SCI-related targets were collected. Then, 4 core nodes (ALB, AKT1, MAPK1, and EGFR) were discerned via construction of a PPI network of 111 common targets. The KEGG enrichment analysis results indicated that the Ras signaling pathway, estrogen signaling pathway, and vascular endothelial growth factor (VEGF) signaling pathway were enriched in the development of SCI. The results of molecular docking demonstrated that the effects of CR have a strong affinity with the 4 pivotal targets. Experimental validation in a rat model showed that CR could effectively improve the recovery of motor function and mechanical pain threshold after SCI.

Conclusions: In summary, it revealed the mechanism of CR treatment for SCI involve active ingredients, targets and signaling pathways, providing a scientific basis for future investigations into the mechanism underlying CR treating for SCI.

Keywords: Spinal cord injury (SCI); Chuanxiong Rhizoma (CR); network pharmacology; molecular docking

Submitted Apr 20, 2021. Accepted for publication Jul 13, 2021.

doi: 10.21037/atm-21-2529

View this article at: https://dx.doi.org/10.21037/atm-21-2529 


\section{Introduction}

Spinal cord injury (SCI) is one of the most severe traumatic diseases causing permanent disability such as functional loss and neurological dysfunction (1-3). It can be divided into primary injury and secondary injury phases on the basis of its complex pathophysiology. Primary SCI is transient and mainly caused by a direct compression and local bleeding. After primary injury, the spinal cord pathophysiology changes are mainly reflected by impaired blood circulation, and tearing of nerve cells and axon membranes, which releases harmful inflammatory mediators and eventually triggers secondary injury. Secondary injury is an initiative process that includes a series of pathological events such as oxidative stress, hemorrhage, neuron apoptosis, excitotoxicity, and posttraumatic inflammation (4).

Neuroinflammation, ischemia, and hypoxia following SCI play a crucial role in the development of SCI (5). Previous studies have shown that inhibition of the inflammatory response can decrease the expression of inflammationrelated factors, thereby reducing secondary damage caused by inflammation and creating a favorable microenvironment for axonal regeneration $(6,7)$. Ameliorating the hypoxic ischemic microenvironment of the local spinal cord could reduce the secondary damage and improve the recovery of neurological function (8).

Network pharmacology is a promising method that uses bioinformatics and pharmacology to uncover the interactions of traditional Chinese medicine (TCM) on diseases, providing a new technical route and logical guidance for understanding and developing of drugs in a visual way $(9,10)$. Chuanxiong Rhizoma (CR), the dried rhizomes of Ligusticum striatum DC, is a traditional Chinese medical herb widely applied for the therapy of brain and heart diseases, such as stroke and coronary heart disease $(11,12)$. Recent pharmacological studies have demonstrated that there are many effective ingredients in CR, such as ferulic acid, senkyunolide I, and ligustilide, and that it has multiple pharmacological effects including anti-inflammatory, anti-oxidative, and anti-obesity $(13,14)$; however, how CR exerts therapeutics effects on SCI remains unclear.

In this study, we first time used the network pharmacology approach as a tool to explore the active ingredients of CR and investigate its underlying mechanism of action in the treatment of SCI. Then, molecular docking of hub targets was used to verify network pharmacology of selected active components. Finally, we validated the neuroprotection role of CR and the key targets expression in an SCI-rat model. The analysis workflow of this research is shown in Figure 1. We present the following article in accordance with the ARRIVE reporting checklist (available at https://dx.doi.org/10.21037/ atm-21-2529).

\section{Methods}

\section{Acquiring of pharmacodynamic ingredients and related targets in $C R$}

The TCMSP database is a systematic online pharmacological database which is used for selecting compounds and evaluating the pharmacokinetic effectiveness of TCM (15). Using the TCMSP database, we obtained active constituents of $\mathrm{CR}$ at the thresholds: oral bioavailability $(\mathrm{OB}) \geq 30 \%$ and drug-likeness $(\mathrm{DL}) \geq 0.18$. The chemical structures were retrieved from PubChem (16). The SwissTargetPrediction online database and PharMapper were used to identify the gene targets corresponding to chemical structures for active compounds.

\section{Potential target genes for SCI}

The action targets related to SCI were retrieved from the Therapeutic Target Database (TTD) and GeneCards $(17,18)$. All the collected genes of CR and SCI were merged before being rectified by the Uniprot database (19). Then, common target genes were obtained using a Venn diagram. The study was in accordance with the Declaration of Helsinki (as revised in 2013).

\section{Construction of the candidate target protein-protein interaction (PPI) network of CR in treating SCI}

To explore the interaction mechanism of these common targets, we putted theses genes into Search Tool for the Retrieval of Interacting Genes/Proteins (STRING) database 11.0 to build a PPI network (20). The candidate target genes were uploaded to the STRING database to visualize and establish the PPI network set the parameter as interaction value $>0.9(21)$.

\section{Gene ontology (GO) and Kyoto Encyclopedia of Genes and Genomes (KEGG) patbway enrichment analysis}

In order to investigate the GO BP and the contributing signaling pathway of the target genes in the treatment of SCI, GO enrichment analysis and KEGG pathway annotation 


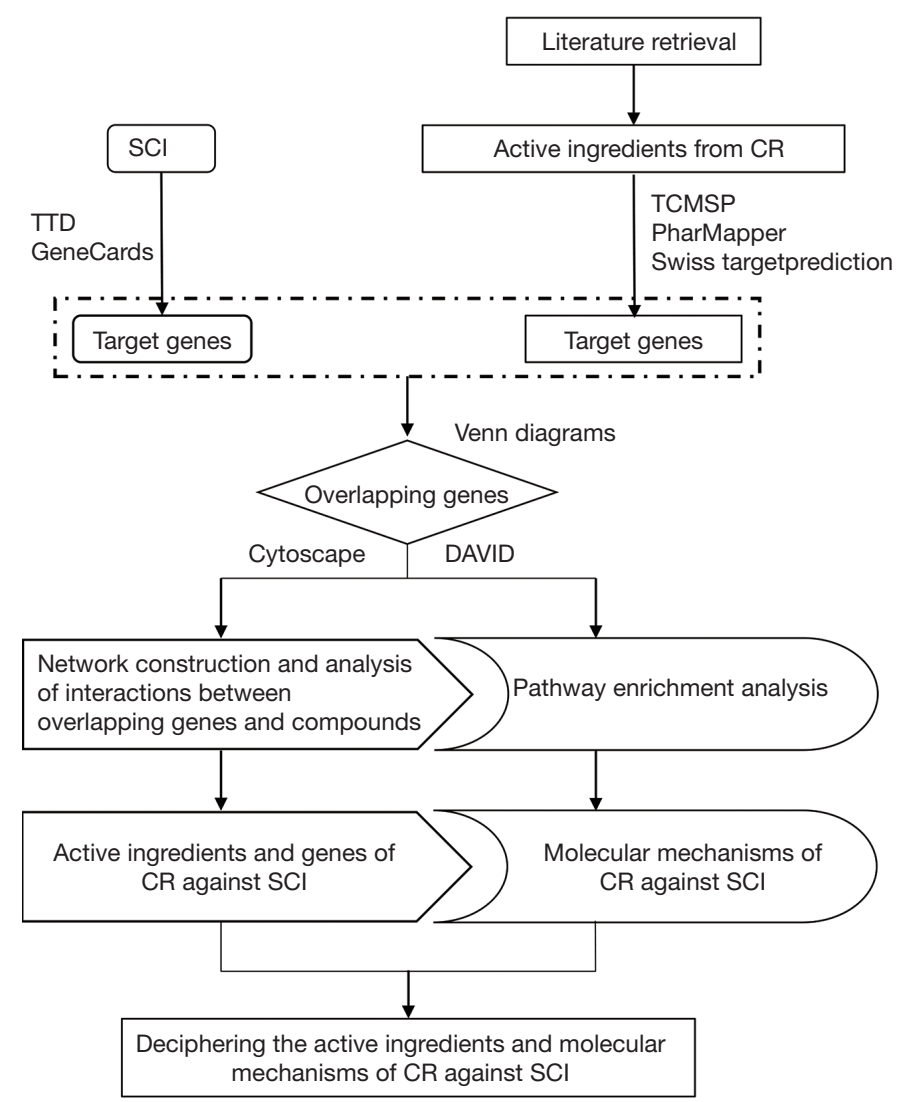

Figure 1 The analysis program flowchart of the study. CR, Chuanxiong Rhizoma; SCI, spinal cord injury; TTD, Therapeutic Target Database.

were performed using the package "ClusterProfifiler" (22). Adjusted $\mathrm{P}$ value $<0.05$ was considered statistically significant.

\section{Molecular docking technology}

The three-dimensional (3D) tructures of the proteins were downloaded from the Research Collaboratory for Structural Bioinformatics (RCSB) Protein Data Bank (PDB) databases. AutodockTools 1.5.6 was performed to dcok the receptor protein with the selective molecule ligands of the active ingredients of CR.

Molecular docking studies were conducted by using AutodockTools 1.5.6. For the results of docking, a score of $0-10$ indicates that binding capacity of the protein from weak to strong, which is the negative logarithm of the experimental dissociation/inhibition constant (pKd/pKi) (23).

\section{Animals}

Adult female Sprague Dawley rats weighing $250 \pm 25 \mathrm{~g}$ (10-week-old) were obtained from Gempharmatech Co. Ltd. and were housed in a humidity- and temperaturecontrolled environment. The experimental processes were approved by the Ethics Committee of Tianjin Institute of Radiation Medicine (IRM-DWLL-2019039) and according to the National Institutes of Health in the Guide for the Care and Use of Laboratory Animals (NIH Publications No. 85-23, revised 1996).

\section{Experimental design}

The rats were firstly randomly assigned to 3 groups: SCI only, Sham group, and SCI $+1.8 \mathrm{~g} / \mathrm{kg} \mathrm{CR}$ (rats were orally administered CR daily after SCI). Each group had 10 rats. Intervention doses of CR were determined based on previous studies (24).

\section{$S C I$}

The contusion SCI model was established using a New 
Table 1 The primers of RT-PCR assays

\begin{tabular}{lll}
\hline & Forward primer & Reverse primer \\
\hline ALB & GAACTATGCTGAGGCCAAGGATGTC & GCTAACACCTGCTAACACCTGGAC \\
AKT1 & TGTGTCCTGCCTCCCTCTTTCC & TACTCACTGGTGCCTCAAGGTCTAG \\
MAPK1 & GGTTGGGTTTGGGTTTGTTCATTG & CGCTGCCTTCTGCTGCTTCTAC \\
EGFR & GACCTCCTGAGTTGCCTTTGATGG & TCCCTCCTCTCTCACTCGCATTG \\
\hline
\end{tabular}

Table $2 \mathrm{OB}$ and DL values of the 7 active compounds of CR Molecule ID

\begin{tabular}{llll}
\hline & Molecule name & OB (\%) & DL \\
\hline MOL002140 & Perlolyrine & 65.95 & 0.27 \\
MOL000433 & FA & 68.96 & 0.71 \\
MOL002151 & Senkyunone & 47.66 & 0.24 \\
MOL002157 & Wallichilide & 42.31 & 0.71 \\
MOL001494 & Mandenol & 42 & 0.19 \\
MOL002135 & Myricanone & 40.6 & 0.51 \\
MOL000359 & Sitosterol & 36.91 & 0.75 \\
\hline
\end{tabular}

$\mathrm{OB}$, oral bioavailability; DL, drug-likeness; CR, Chuanxiong Rhizoma.

York University Impactor device (NYU, New York, USA) as previously described (25). In the sham group, the rats underwent laminectomy only.

\section{Locomotion recovery analysis}

Basso Beattie Bresnahan (BBB) score and inclined plane test were performed prior to test the locomator function of rat after SCI. The BBB test score which evaluated the movement of lower limbs on a scale of 0 to 21 . Scoring was based on the accurate observation of hindlimb movement during a $5 \mathrm{~min}$ in the open field. The maximum angle that a rat could stay on an angle plane for $5 \mathrm{~s}$ without falling was recorded. Each test was performed in triplicate. This testing was performed weekly until 4 weeks post-SCI.

\section{Mechanical allodynia}

To evaluate the nerve function recovery of rats after SCI, the pain nociception was measured using the automated von Frey filaments. After at least $30 \mathrm{~min}$ of habituated the environment, a touch stimulator was applied to the center of plantar surface of each hind paw using a adjusted angled mirror. The paw with threshold was calculated as an average of hind paws at the interval of $10 \mathrm{~s}$.

\section{Quantitative real-time polymerase chain reaction (qRT-PCR) verification}

The procedure was performed as previously described (26). Total RNA was isolated from the spinal cord tissues with TRIZOL reagent (Invitrogen, Shanghai, China), and reverse transcription was then used with a poly(T) adapter (Takara, Dalian, China) into cDNA following the manufacturer's instructions. Real-time quantitative PCR was performed using SYBR green Premix Ex TaqTM Kit (Takara, Dalian, China). The primers as listed in Table 1.

\section{Statistical analysis}

All data were shown as mean \pm standard error of the mean (SEM). GraphPad Prism 6.0 (GraphPad Software, Inc., La Jolla, CA, USA) was used for statistical analysis and image construction. Multiple different groups were analyzed with a one-way analysis of variance (ANOVA) followed by Bonferroni test. A $\mathrm{P}$ value $<0.05$ was considered statistically significant.

\section{Results}

Screening of active ingredients and potential targets in $C R$

As results, 7 chemical components of CR were collected from the TCMSP database (Table 2). Furthermore, based on the structure similarity algorithm and data mining, we finally obtained 246 potential targets of CR and 1,260 SCIrelated targets. Ultimately, by taking an intersection of the CR-target genes and SCI-related target genes, 111 common targets were summarized (Figure $2 A$ ). We then established a compound-target network of CR (Figure 2B). 
A

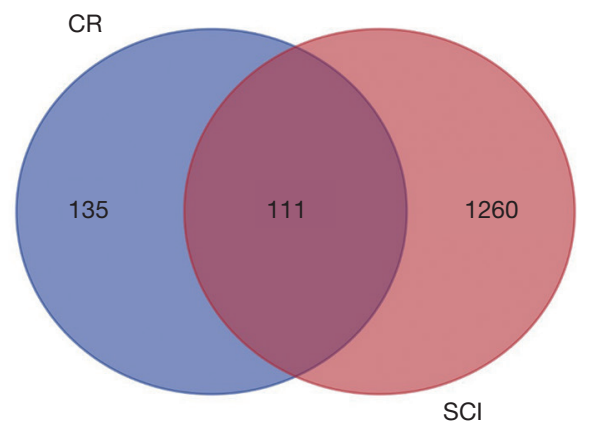

B

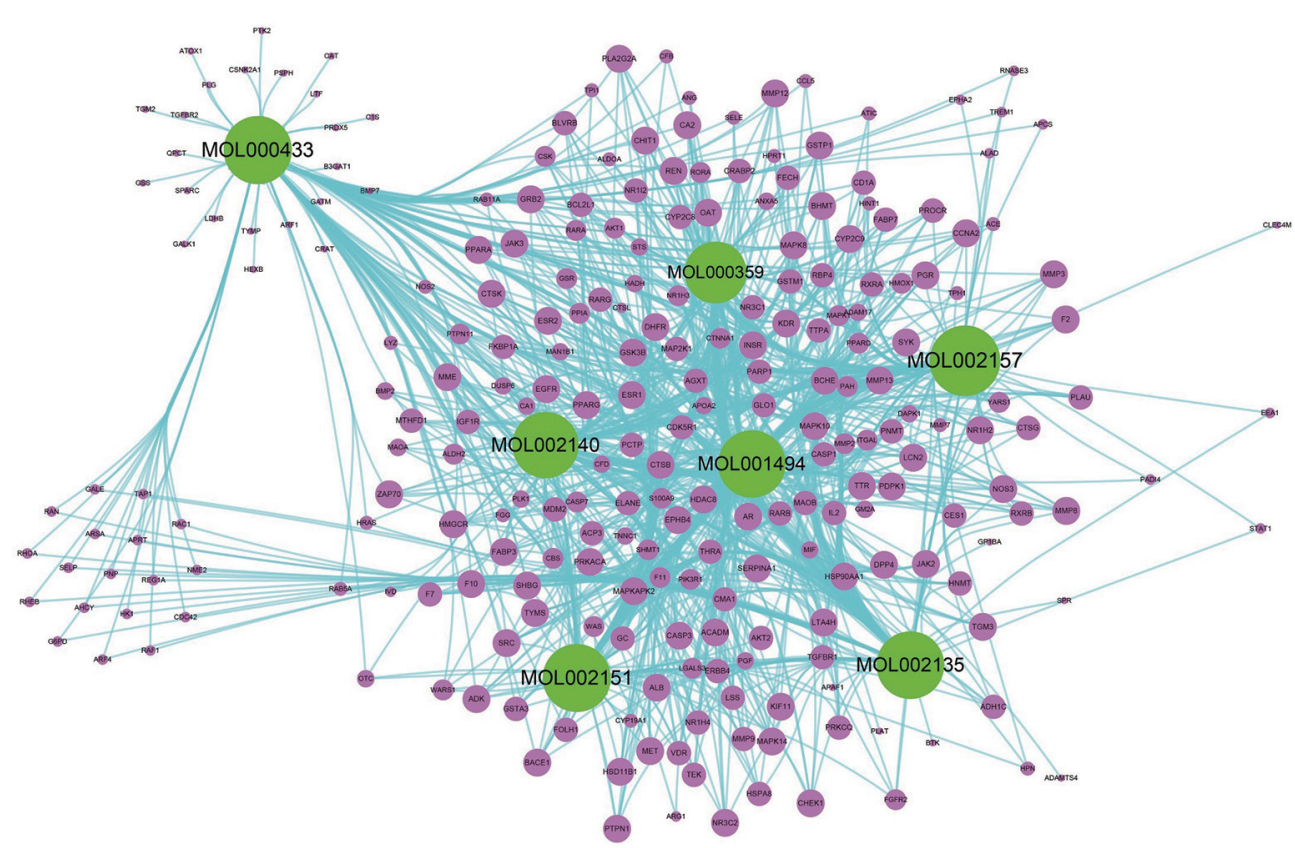

Figure 2 Venn analysis and network analysis of active compounds and potential targets of CR in SCI. (A) The Venn diagram of target proteins of CR and SCI-related targets based on open targets platform. (B) The compounds-targets network diagram of CR to treat SCI, square nodes represent compounds, circular nodes represent target proteins. CR, Chuanxiong Rhizoma; SCI, spinal cord injury.

\section{The PPI network against SCI}

A PPI network showed that the proteins encoded by the 111 common targets had complex interactions (Figure 3). The network contained 109 nodes and 1,229 edges; which were the core targets of CR in the treatment of SCI. The node color showed the degree of node contribution in the network and if there was a positive correlation in the development of SCI. Among them ALB, AKT1, MAPK1, EGFR, MMP9, CASP3, MAPK8, SRC, HRAS, HSP90AA1, CAT, and MMP2 were significantly enriched, which were considered to be hub genes. The top 10 genes in the network were ranked by degrees (Table 3). Comparing the results, ALB, AKT1, MAPK1, and EGFR were selected to conduct molecular docking experiments.

\section{GO and KEGG pathway enrichment analyze}

The 111 potential targets of CR to treat SCI were further analyzed for BP and KEGG pathways. It was revealed that BP with high enrichment scores, such as response to steroid hormone, neutrophil mediated immunity, and neutrophil activation and degranulation (Figure 4A). The KEGG analysis results showed the main pathway included 


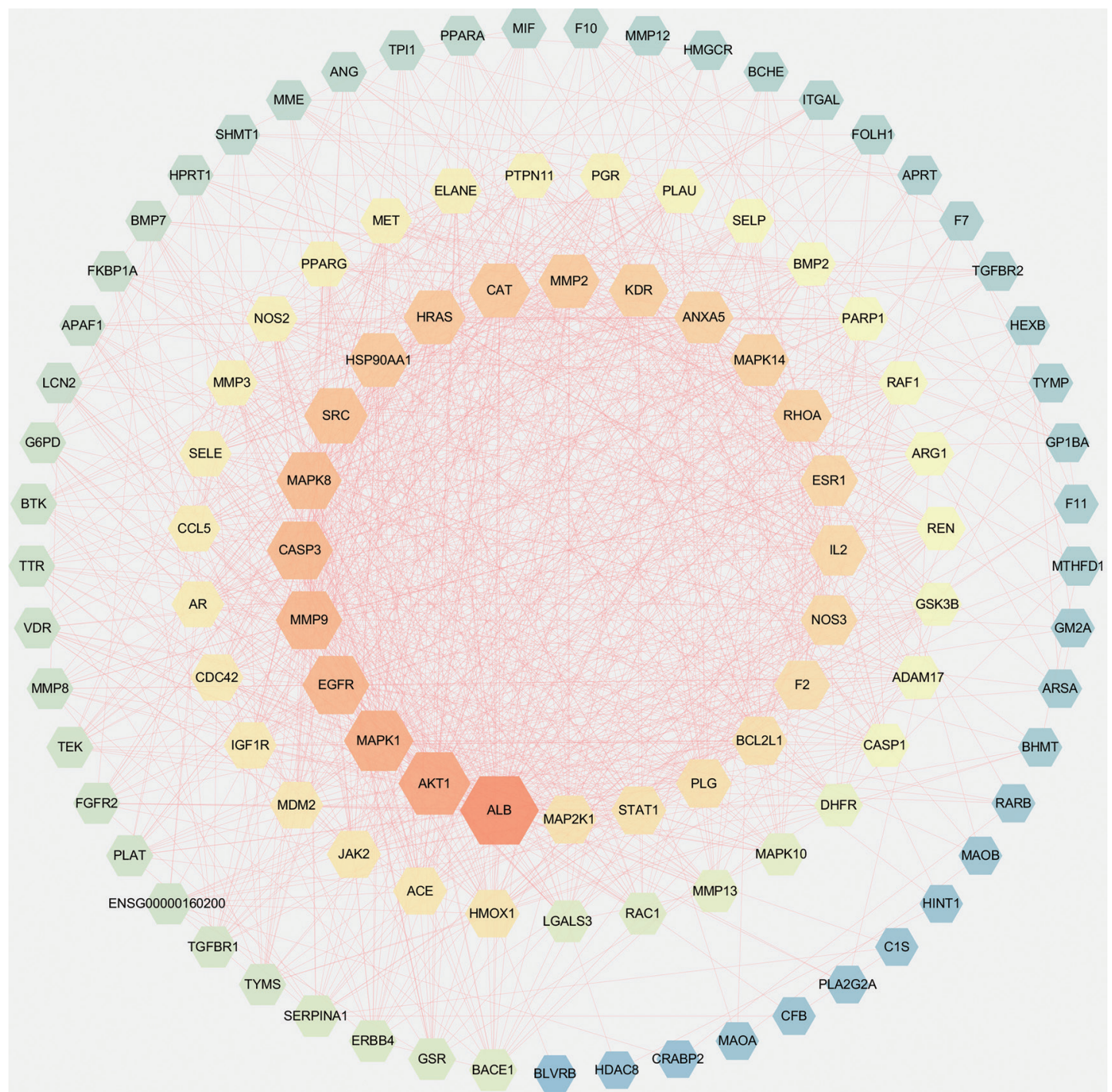

Figure 3 The PPI network of CR target genes. The PPI network contains 109 nodes and 1,229 edges. PPI, protein-protein interaction; CR, Chuanxiong Rhizoma.

the estrogen signaling pathway, Ras signaling pathway and vascular endothelial growth factor (VEGF) signaling pathway were most closely related to SCI (Figure 4B).

\section{Molecular docking of active compounds and bub proteins}

The molecular docking results showed that there was a good binding between all the 7 active compounds of CR could easily bind the following selected proteins (Figure 5): ALB, AKT1, MAPK1, and EGFR. At the same time, the result of molecular docking verified the reliability of network pharmacology. The docking results are shown in
Table 4.

\section{CR improved the functional recovery and mechanical allodynia of rats after $S C I$}

To examine the efficacy of CR in SCI, the motor function recovery of SCI rats were evaluated by using BBB score and mechanical allodynia were applied from day 1 until 4 weeks post-SCI. There was a significant difference in the mean $\mathrm{BBB}$ score and incline plane test between the Injury and CR-treated group after 1 week post-injury, which showed that CR could improve functional recovery after SCI 
Table 3 Top 10 nodes in the network ranked by degree

\begin{tabular}{llc}
\hline Rank & Name & Score \\
\hline 1 & ALB & 83 \\
2 & AKT1 & 69 \\
3 & MAPK1 & 64 \\
4 & EGFR & 59 \\
5 & MMP9 & 58 \\
6 & CASP3 & 57 \\
7 & MAPK8 & 55 \\
8 & SRC & 52 \\
9 & CAT & 48 \\
\hline
\end{tabular}

(Figure $6 A, B)$. Meanwhile, CR could also improve mechanical pain threshold (Figure 6C).

\section{In vivo experiment}

In order to clarify the mechanism of CR on Spinal cord injury rats, we validated the expression of the four core genes by using qRT-PCR method. Compared with SCI groups, the expression of ALB, AKT1, MAPK1, EGFR with CR treatment group has decreased, which was consistent with above results. The results were shown in Figure 7 .

\section{Discussion}

In recent times, increasing attention has been paid to research focused on natural products $(27,28)$. Through the combination of network pharmacology and molecular biology network analysis, several new therapeutic drugs derived from natural products have been decoded (29). This approach provides new insight into the systemic linkages between different diseases and therapeutic targets as a whole, and provides a promising and powerful tool to elucidating disease mechanisms at a systemic level and facilitate the discovery of potential active compounds (30). Recently, many Traditional Chinese Medicine formulae have been proven to be effective in treating diseases. The network pharmacology method was used to screen and confirm the traditional Chinese medicine components in the formulation of TCM. Tao et al. identified potential targets of Coronavirus disease (COVID-19) through network pharmacology and obtained mainly 168 pathways related to COVID-19. This study confirmed the mechanism of Huashi Baidu formula (HSBDF) in treating COVID-19 and indirectly proved the effectiveness of Huashi Baidu formula (HSBDF). TNF signaling pathway, PI3K-Akt signaling pathway, NOD-like receptor signaling pathway, MAPK signaling pathway, and HIF-1 signaling pathway, and other signaling pathways were also found to be connected (31). $\mathrm{Gu} e t \mathrm{al}$. collected the components of indigo naturalis (IN) and predicted their targets by using network pharmacology and obtained the ulcerative colitis targets in GEO database at the same time. This research concluded that IN could regulate genes and biological processes related to regulation of reactive oxygen species and cell proliferation and the participation of apoptosis (32).

In this research, the network pharmacology approach and molecular docking experiments were used to reveal the bioactive ingredients of CR and its molecular mechanism in the treatment of SCI. There were 7 bioactive ingredients and 111 different targets identified via the online database. We established a PPI network of candidate targets which revealed 109 nodes and 1,229 edges. Through the degree values, it was revealed that ALB, AKT1, MAPK1, and EGFR proteins were likely to play key roles in the development of SCI.

In addition, on the basis of GO enrichment analysis, the BP terms enriched in target genes mainly focused on the regulation of neutrophil activation, neutrophil degranulation, and steroid hormone mediated signaling pathway. The KEGG enrichment analysis indicated that 111 target proteins were significantly enriched in the estrogen signaling pathway, VEGF signaling pathway, and Ras signaling pathway, which are closely related to the signal pathway of SCI.

A previous study demonstrated that up-regulation of the Ras signaling pathway aggravates the development of SCI by damaging spinal cord-injured neurons regeneration and causing neural microenvironment imbalances, and that inhibiting this signal pathways could restore the injured neuronal cell migration, adhesion, neurogenesis, and dendritic spine development (33). A characteristic of SCI is an inflammatory response that induces the increased expression of several cytokines, estrogen has been reported have an interfering effect and it reduces the expression of several inflammasome components after SCI $(34,35)$. The VEGF represents an endothelial-specific growth factor which plays an important role in vasculogenesis and angiogenesis. In the nervous system, it has been 


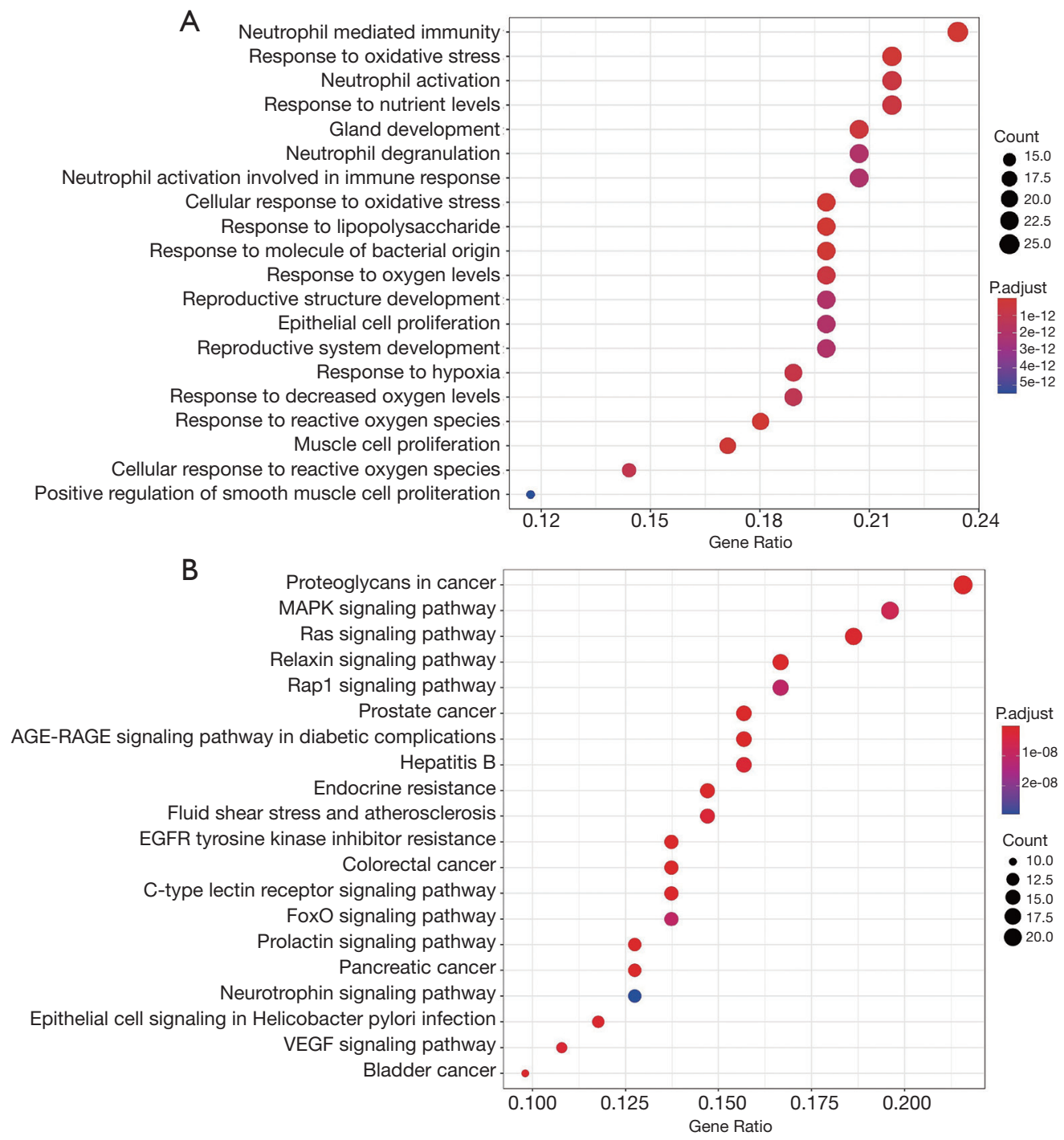

Figure 4 The GO and KEGG enrichment analysis of core targets of CR in treating SCI. (A) The top 20 GO biological process analysis. (B) The top 20 KEGG signal pathways annotation. GO, Gene Ontology; BP, biological process; KEGG, Kyoto Encyclopedia of Genes and Genomes; CR, Chuanxiong Rhizoma; SCI, spinal cord injury.

demonstrated that VEGF exerts a neuroprotective effect to promote nerve regeneration and stimulate the growth and survival of neurons by upregulating MAP2 expression (36-39). Chen et al. demonstrated that the VEGF signaling pathway plays a vital role in SCI secondary injury; activation of the VEGF signaling pathway induced the VEGF protein expression to increase, it can improve the local hypoxic microcirculation environment, reduce SCI secondary damage, and promote the improvement of locomotor function (8).

To confirm the above results of network pharmacology analysis, we also conducted an SCI rat model animal validation experiment, the results of which showed that CR can effectively promote locomotion recovery. These results 

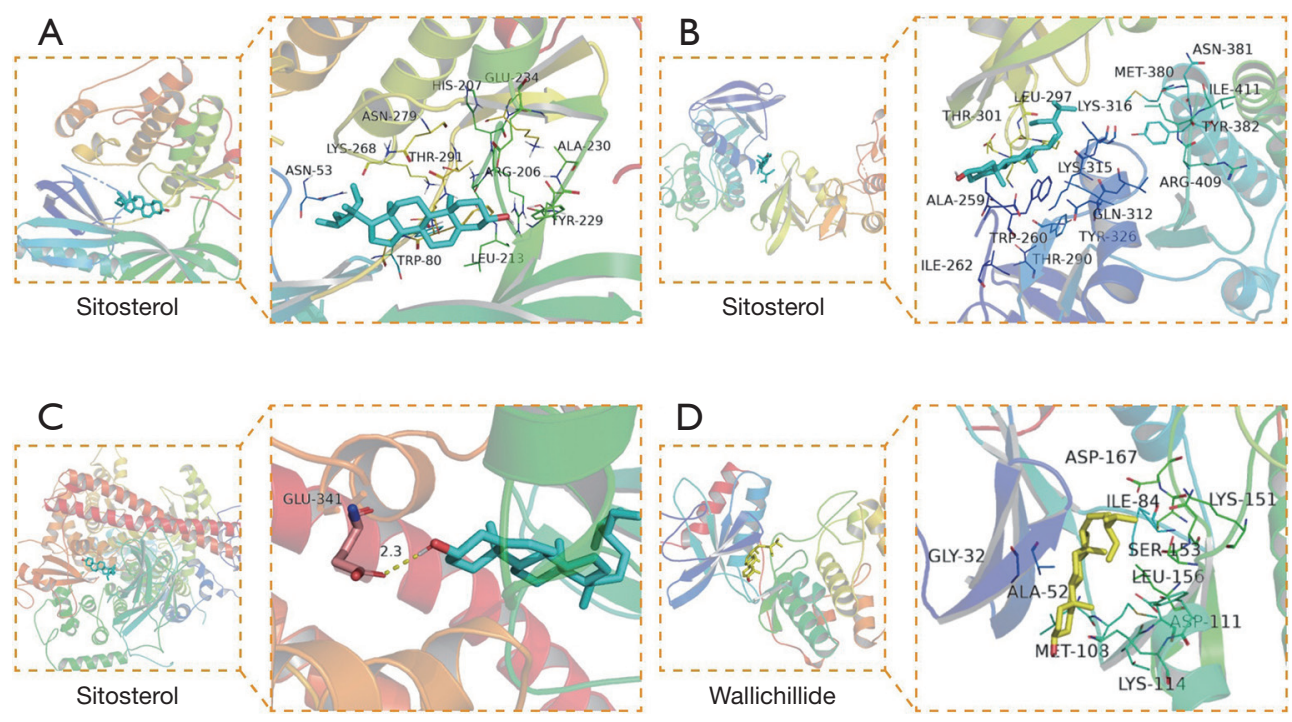

Figure 5 The docking of 4 core targets and their strongest binding components of CR. (A) ALB, (B) AKT1, (C) MAPK1, (D) EGFR. CR, Chuanxiong Rhizoma.

Table 4 The binding energy of CR with the target proteins (kcal/mol)

\begin{tabular}{lcccc}
\hline Receptor ligand & ALB & AKT1 & MAPK1 & EGFR \\
\hline Sitosterol & -11 & -8.4 & -8.7 & -7.3 \\
Mandenol & -6.8 & -5.5 & -5.6 & -4.7 \\
Myricanone & -8.6 & -8.2 & -7.7 & -7.0 \\
Perlolyrine & -9.6 & -8.0 & -7.8 & -7.3 \\
Senkyunone & -9.0 & -7.2 & -6.8 & -67.2 \\
Wallichilide & -7.1 & -6.7 & -5.8 & -8.7 \\
FA & -9.8 & -7.7 & -8.1 & -5.9 \\
\hline
\end{tabular}

CR, Chuanxiong Rhizoma; FA, faradiol. indicated that through the above mechanisms, CR is closely associated with these key target proteins, $\mathrm{BP}$, and signaling pathways against SCI. Further experiments and clinical validations are still required to verify our findings.

\section{Conclusions}

In summary, we first elucidated the pharmacological effects of CR on SCI through network pharmacology analysis. A total of 111 target genes including ALB, AKT1, MAPK1, and EGFR were the key target proteins of CR anti-SCI. GO and KEGG Functional enrichment showed that proteins targeted by CR were mainly enriched in estrogen 

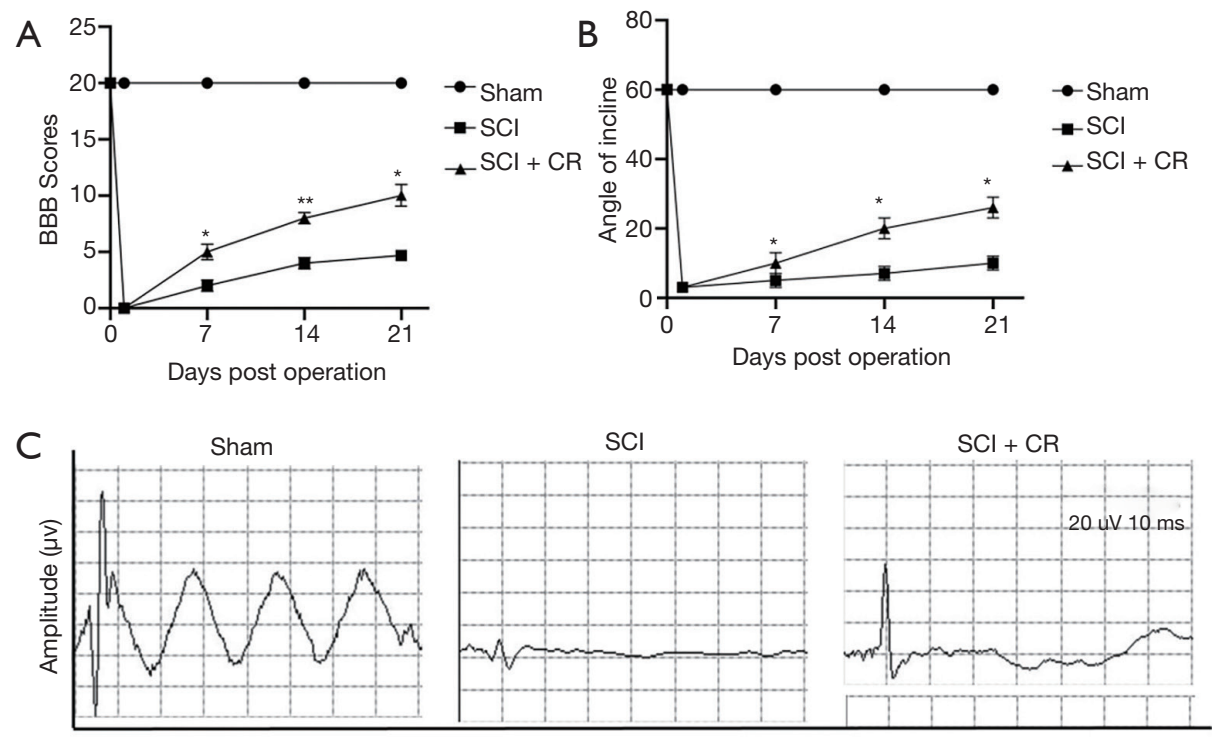

Latancy (ms)

Figure 6 CR promotes recovery of locomotor function and mechanical allodynia in SCI rats. (A,B) The BBB locomotor scores and inclined plane test at $0,7,14$, and $21 \mathrm{~d}$ post-SCI were shown for all groups of rats. (C) MEP were tested at 4 weeks for all groups of rats. * $\mathrm{P}<0.05$; **, $\mathrm{P}<0.01$. CR, Chuanxiong Rhizoma; SCI, spinal cord injury; BBB, Basso Beattie Bresnahan; MEP, motor evoked potential.

A
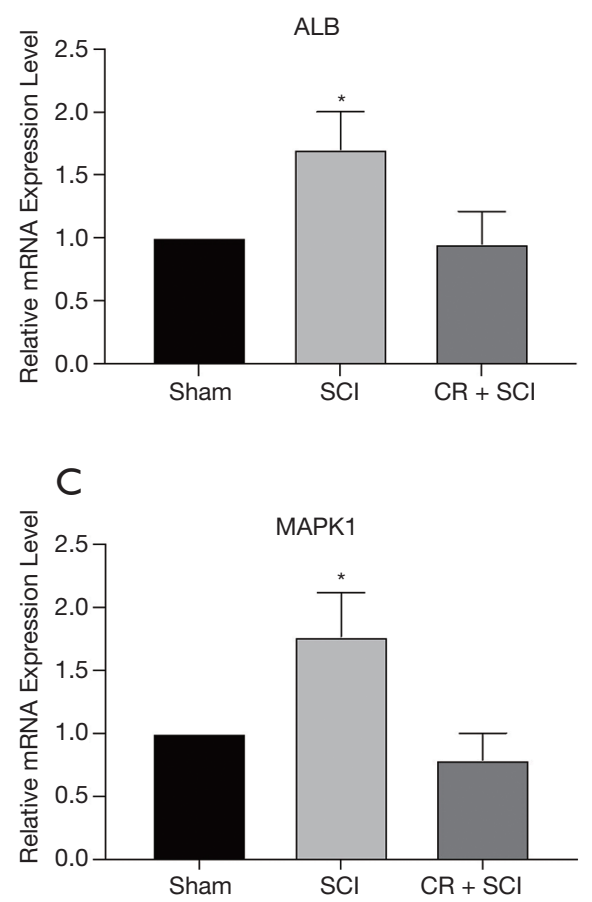

B
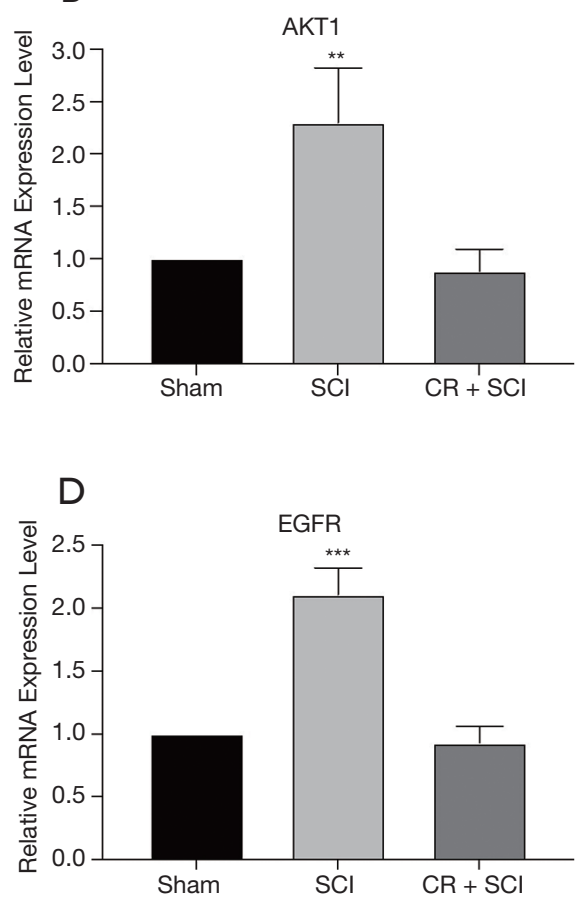

Figure 7 The expression of four core genes in SCI rats after 4 weeks of CR treatment. qRT-PCR was used to verify the genes (A) ALB, (B) AKT1, (C) MAPK1, (D) EGFR. * indicated $\mathrm{P}<0.05$, ** indicated $\mathrm{P}<0.01$, *** indicated $\mathrm{P}<0.001$. SCI, spinal cord injury; CR, Chuanxiong Rhizoma; qRT-PCR, quantitative real-time polymerase chain reaction. 
signaling pathway, Ras signaling pathway, and VEGF signaling pathway. This research may provide a theoretical basis for further studies of CR in SCI.

\section{Acknowledgments}

Funding: This study was supported by grants from the National Key Research and Development Project of Stem Cell and Transformation Research (2019YFA0112100), NSFC programs (81620108018, 81930070), Tianjin Key Research and Development Plan, Key Projects for Science and Technology Support (19YFZCSY00660), and Tianjin Research Innovation Project for Postgraduate Students (2019YJSB106).

\section{Footnote}

Reporting Checklist: The authors have completed the ARRIVE reporting checklist. Available at https://dx.doi. org/10.21037/atm-21-2529

Data Sharing Statement: Available at https://dx.doi. org/10.21037/atm-21-2529

Conflicts of Interest: All authors have completed the ICMJE uniform disclosure form (available at https://dx.doi. org/10.21037/atm-21-2529). The authors have no conflicts of interest to declare.

Ethical Statement: The authors are accountable for all aspects of the work in ensuring that questions related to the accuracy or integrity of any part of the work are appropriately investigated and resolved. The experimental processes were approved by the Ethics Committee of Tianjin Institute of Radiation Medicine (IRMDWLL-2019039) and according to the National Institutes of Health in the Guide for the Care and Use of Laboratory Animals (NIH Publications No. 85-23, revised 1996). The study was in accordance with the Declaration of Helsinki (as revised in 2013).

Open Access Statement: This is an Open Access article distributed in accordance with the Creative Commons Attribution-NonCommercial-NoDerivs 4.0 International License (CC BY-NC-ND 4.0), which permits the noncommercial replication and distribution of the article with the strict proviso that no changes or edits are made and the original work is properly cited (including links to both the formal publication through the relevant DOI and the license). See: https://creativecommons.org/licenses/by-nc-nd/4.0/.

\section{References}

1. Niclis JC, Turner C, Durnall J, et al. Long-Distance Axonal Growth and Protracted Functional Maturation of Neurons Derived from Human Induced Pluripotent Stem Cells After Intracerebral Transplantation. Stem Cells Transl Med 2017;6:1547-56.

2. Sharwood LN, Wiseman T, Tseris E, et al. Pre-existing mental disorder, clinical profile, inpatient services and costs in people hospitalised following traumatic spinal injury: a whole population record linkage study. Inj Prev 2020. [Epub ahead of print]. doi: 10.1136/injuryprev-2019-043567.

3. Fakhoury M. Spinal cord injury: overview of experimental approaches used to restore locomotor activity. Rev Neurosci 2015;26:397-405.

4. Silva NA, Sousa N, Reis RL, et al. From basics to clinical: a comprehensive review on spinal cord injury. Prog Neurobiol 2014;114:25-57.

5. Gong L, Lv Y, Li S, et al. Changes in transcriptome profiling during the acute/subacute phases of contusional spinal cord injury in rats. Ann Transl Med 2020;8:1682.

6. Mortezaee K, Khanlarkhani N, Beyer C, et al. Inflammasome: Its role in traumatic brain and spinal cord injury. J Cell Physiol 2018;233:5160-9.

7. Dai $W$, Wang $X$, Teng H, et al. Celastrol inhibits microglial pyroptosis and attenuates inflammatory reaction in acute spinal cord injury rats. Int Immunopharmacol 2019;66:215-23.

8. Chen H, Li J, Liang S, et al. Effect of hypoxia-inducible factor-1/vascular endothelial growth factor signaling pathway on spinal cord injury in rats. Exp Ther Med 2017;13:861-6.

9. Chandran U, Mehendale N, Tillu G, et al. Network Pharmacology of Ayurveda Formulation Triphala with Special Reference to Anti-Cancer Property. Comb Chem High Throughput Screen 2015;18:846-54.

10. Chen L, Lv D, Wang D, et al. A novel strategy of profiling the mechanism of herbal medicines by combining network pharmacology with plasma concentration determination and affinity constant measurement. Mol Biosyst 2016;12:3347-56.

11. Pu ZH, Dai M, Xiong L, et al. Total alkaloids from the rhizomes of Ligusticum striatum: a review of chemical analysis and pharmacological activities. Nat Prod Res 2020. [Epub ahead of print]. doi: 
10.1080/14786419.2020.1830398.

12. Ho JW, Cheung MW, Yu VW. Active phytochemicals from Chinese herbs as therapeutic agents for the heart. Cardiovasc Hematol Agents Med Chem 2012;10:251-5.

13. Liu ZK, Ng CF, Shiu HT, et al. A traditional Chinese formula composed of Chuanxiong Rhizoma and Gastrodiae Rhizoma (Da Chuanxiong Formula) suppresses inflammatory response in LPS -induced RAW 264.7 cells through inhibition of NF-kappaB pathway. J Ethnopharmacol 2017;196:20-8.

14. Liu Q, Zhou J, Yu J, et al. Systematic and efficient separation of 11 compounds from Rhizoma Chuanxiong via counter-current chromatography-solid phase extraction-counter-current chromatography hyphenation. J Chromatogr A 2014;1364:204-13.

15. Ru J, Li P, Wang J, et al. TCMSP: a database of systems pharmacology for drug discovery from herbal medicines. J Cheminform 2014;6:13.

16. Kim S, Thiessen PA, Bolton EE, et al. PubChem Substance and Compound databases. Nucleic Acids Res 2016;44:D1202-13.

17. Stelzer G, Rosen N, Plaschkes I, et al. The GeneCards Suite: From Gene Data Mining to Disease Genome Sequence Analyses. Curr Protoc Bioinformatics 2016;54:1.30.1-1.30.33.

18. Wang Y, Zhang S, Li F, et al. Therapeutic target database 2020: enriched resource for facilitating research and early development of targeted therapeutics. Nucleic Acids Res 2020;48:D1031-41.

19. UniProt Consortium T. UniProt: the universal protein knowledgebase. Nucleic Acids Res 2018;46:2699.

20. Szklarczyk D, Morris JH, Cook H, et al. The STRING database in 2017: quality-controlled protein-protein association networks, made broadly accessible. Nucleic Acids Res 2017;45:D362-8.

21. Merico D, Isserlin R, Stueker O, et al. Enrichment map: a network-based method for gene-set enrichment visualization and interpretation. PLoS One 2010;5:e13984.

22. Yu G, Wang LG, Han Y, et al. clusterProfiler: an R package for comparing biological themes among gene clusters. OMICS 2012;16:284-7.

23. Hsin KY, Ghosh S, Kitano H. Combining machine learning systems and multiple docking simulation packages to improve docking prediction reliability for network pharmacology. PLoS One 2013;8:e83922.

24. Wang M, Yao M, Liu J, et al. Ligusticum chuanxiong exerts neuroprotection by promoting adult neurogenesis and inhibiting inflammation in the hippocampus of ME cerebral ischemia rats. J Ethnopharmacol 2020;249:112385.

25. Cheriyan T, Ryan DJ, Weinreb JH, et al. Spinal cord injury models: a review. Spinal Cord 2014;52:588-95.

26. Yi J, Tian M, Hu L, et al. The mechanisms of celastrol in treating papillary thyroid carcinoma based on network pharmacology and experiment verification. Ann Transl Med 2021;9:866.

27. Hiebl V, Ladurner A, Latkolik S, et al. Natural products as modulators of the nuclear receptors and metabolic sensors LXR, FXR and RXR. Biotechnol Adv 2018;36:1657-98.

28. Liang JW, Wang MY, Olounfeh KM, et al. Network pharmacology-based identifcation of potential targets of the flower of Trollius chinensis Bunge acting on antiinflammatory effectss. Sci Rep 2019;9:8109.

29. Li XJ, Jiang ZZ, Zhang LY. Triptolide: progress on research in pharmacodynamics and toxicology. J Ethnopharmacol 2014;155:67-79.

30. Huang J, Cheung F, Tan HY, et al. Identification of the active compounds and significant pathways of yinchenhao decoction based on network pharmacology. Mol Med Rep 2017;16:4583-92.

31. Tao Q, Du J, Li X, et al. Network pharmacology and molecular docking analysis on molecular targets and mechanisms of Huashi Baidu formula in the treatment of COVID-19. Drug Dev Ind Pharm 2020;46:1345-53.

32. Gu S, Xue Y, Gao Y, et al. Mechanisms of indigo naturalis on treating ulcerative colitis explored by GEO gene chips combined with network pharmacology and molecular docking. Sci Rep 2020;10:15204.

33. Xu D, Cao F, Sun S, et al. Inhibition of the Ras/ Raf/ERK1/2 Signaling Pathway Restores Cultured Spinal Cord-Injured Neuronal Migration, Adhesion, and Dendritic Spine Development. Neurochem Res 2016;41:2086-96.

34. Sribnick EA, Wingrave JM, Matzelle DD, et al. Estrogen attenuated markers of inflammation and decreased lesion volume in acute spinal cord injury in rats. J Neurosci Res 2005;82:283-93.

35. Zendedel A, Monnink F, Hassanzadeh G, et al. Estrogen Attenuates Local Inflammasome Expression and Activation after Spinal Cord Injury. Mol Neurobiol 2018;55:1364-75.

36. Song S, Park JT, Na JY, et al. Early expressions of hypoxia-inducible factor 1alpha and vascular endothelial growth factor increase the neuronal plasticity of activated endogenous neural stem cells after focal cerebral ischemia. Neural Regen Res 2014;9:912-8.

37. Ding XM, Mao BY, Jiang S, et al. Neuroprotective effect 
of exogenous vascular endothelial growth factor on rat spinal cord neurons in vitro hypoxia. Chin Med J (Engl) 2005;118:1644-50.

38. Jin K, Mao XO, Greenberg DA. Vascular endothelial growth factor stimulates neurite outgrowth from cerebral

Cite this article as: Tao B, Wang Q, Cao J, Yasen Y, Ma L, Sun C, Shang J, Feng S. The mechanisms of Chuanxiong Rhizoma in treating spinal cord injury based on network pharmacology and experimental verification. Ann Transl Med 2021;9(14):1145. doi: 10.21037/atm-21-2529 cortical neurons via Rho kinase signaling. J Neurobiol 2006;66:236-42.

39. Rosenstein JM, Krum JM, Ruhrberg C. VEGF in the nervous system. Organogenesis 2010;6:107-14. 